\title{
ESPAÇOS HISTÓRICOS, DEUSES HIERÁRQUICOS - GEOGRAFIAS NEGRAS COMO ABERTURA EPISTÊMICA DIASPÓRICA ${ }^{1}$
}

\author{
Geri Augusto ${ }^{2}$
}

\begin{abstract}
Resumo: Este ensaio fotográfico, parte de um projeto maior intitulado "Espaços históricos, deuses hierárquicos", usa a metodologia da autora de uma caminhada epistêmica para argumentar visual e textualmente a importância do conceito de geografias negras para estudos da África e da diáspora que interrogam e exploram espacialmente, bem como temporalmente, uma série de questões, incluindo histórias e memória pública da escravidão, a estética embutida nas contra-paisagens negras, o lugar da ancestralidade nas religiões afro-diaspóricas, e as manifestações globais do anti-racismo negro contemporâneo.
\end{abstract}

Palavras-chave: Geografias negras. Contra-paisagens negras. Escravidão racial. Geografias sagradas, ancestralidade. Mediterrâneo Negro. Memória pública. Religiões afro-diaspóricas. Violência anti-negra.

\begin{abstract}
This photo essay, part of a larger project entitled "Historical Spaces, Hierarchical Gods, " uses the author's methodology of an epistemic walk to argue visually and textually the importance of the concept of black geographies for studies of Africa and the Diaspora which interrogate and explore spatially as well as temporally a range of issues including histories and public memory of slavery, the aesthetics embedded in black counter-landscapes, the place of ancestrality in Afro-Diasporic religions, and contemporary anti-black racism's global manifestations.
\end{abstract}

Key words: BLACK geographies. Black counter-landscapes. Racial slavery. Sacred geographies. Ancestrality. Black Mediterranean. Public memory. Afrodiasporic religions. Anti-black violence.

\section{Introdução}

Geografias negras, um campo interdisciplinar de rápida evolução, está abrindo novas maneiras de pensar sobre toda uma gama de preocupações, tanto históricas quanto contemporâneas, no estudo da África e

\footnotetext{
${ }^{1}$ Este artigo revisa minha apresentacao original a BRASIL EN TEIAS CULTURAIS: Relações Internacionais sob o crivo da crítica cultural, TEIA BRASIL-AMÉRICA DO NORTE: Mesa 5: AmeriÁfricas: aquilombamentos, antirracismos e reexistências negras nas teias do saber, organizada por Prof. Dra. M. Anoria Oliveira, 9 Outubro 2020.

${ }^{2}$ Doutorada em Educação pela George Washington University, mestrada em politicas públicas pela Harvard Kennedy School; professora de relações públicas e internacionais e de estudos africanos [África e Diáspora], e diretora dos estudos de desenvolvimento, no Instituto Watson da Brown University; geri_augusto@brown.edu
} 
da diáspora Africana, bem como novos horizontes para estudos culturais críticos em países pluralistas marcados pela desigualdade. ${ }^{3}$ Cruzando o espaço com o tempo, e dando lugar a resistência, devires e tradução - modo de pensar frequentemente praticado em sociedades e comunidades africanas e afrodescendentes sob a rubrica de pensamento da encruzilhada - geografias negras lançam luz sobre a multiplicidade de maneiras pelas quais os negros podem se inscrever em espaços públicos onde seus corpos, culturas ou pensamentos são indesejados, ou contradizem versões hegemônicas e fáceis da história. Africanos escravizados e seus descendentes livres e quilombolas construíram arquiteturas paisagísticas que constituem contra-narrativas de suas vidas nas Américas, no sul da África ou nas ilhas do Oceano Índico. Ancestralidade e espiritualidade constituem blocos de construção imateriais de espaços de refúgio e proteção, e das lutas para alcançá-los, que são gravadas sobre a terra e as águas. O artigo que se segue, somente uma pequena parte de um projeto maior de fotografias, ensaios e, eventualmente, uma exposição, intitulada "Espaços históricos, deuses hierárquicos", desenvolve essas afirmações visualmente e, com base nisso, postula um coda de geografias e paisagens negras que convida mais reflexão crítica na intersecção de espaço, raça e cultura.
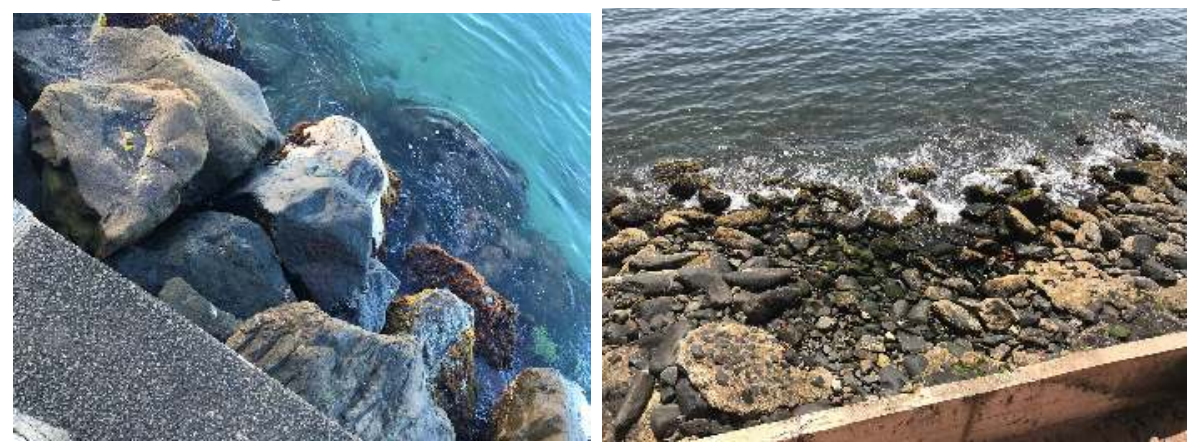

Díptico: Partida / Chegada, Ilha Goree, Senegal, Junho de 2019 e Fort de France, Martinica, Janeiro de 2019.

\footnotetext{
3 Para uma excelente introdução ao campo, em meio a uma literatura em expansão, ver MCKITTRICK, Kathrine \& WOODS, Clyde, (2007), Black geographies and the political of place; BLEDSOE, WRIGHT e EVANS, (2020), "Black Geographies"; e a Edição Especial da Revista da ABPN, (Abr 2020), "Geografias Negras" v. 12.
} 
Uma calmaria enganosamente serena de água sobre pedras, bem na beira do mar, tão parecida na ilha de Gorée quanto em Fort de France, apesar de milhares de quilômetros de distância entre elas. Acho que isso pode ter sido uma memória gêmea duradoura, de corpo e alma, tangível, de muitos africanos e africanas que partiram e chegaram em cativeiro, dos séculos 17 a 19, e por isso preferi em minhas visitas refletir sobre isso. ${ }^{4} \mathrm{Nem}$ a lendária (e contestada) porta-sem-volta, parte do úmido depósito da Casa dos Escravos de Gorée (Maison d'Esclaves ou Maison Pépin), onde humanos e mercadorias eram mantidos, nem o sólido forte de pedra protegendo o tráfego humano que entrava na Martinica, embora essas estruturas fiquem próximas, me interessou muito. Ambos os edifícios são sólidos, desgastados, mas inabaláveis, ainda hoje, a arquitetura dura das Empresas de Senegal, das Ilhas, das Indias e assim por diante. Testemunhos ostentosos, nas duas pontas da jornada horrenda dos cativeiros, sobre a riqueza e os medos dos escravocratas franceses e seus "senhares" euro-africanas, cúmplices ou coagidas, sobre oficiais militares reais, proprietários de plantações/fazendas, comerciantes, financeiros e industriais iniciantes - essas estruturas não contam, de maneiras novas, as histórias que agora me interessam. ${ }^{5}$ Será que a água e as pedras - eternas e comoventes, deslocadas por mãos humanas, mas não por sua criação - também são uma arquitetura de paisagem, que marca a história?

\footnotetext{
${ }^{4}$ As visitas a ambos os locais eram para trabalhar com colegas locais, não para turismo. Tendo vivido e trabalhado na África Austral por 18 anos, quando mais jovem, sempre fui apenas uma turista muito relutante e conflituosa. Para um ensaio incisivo e reflexivo sobre as viagens da diáspora às fortalezasprisão da costa da África Ocidental, consulte ADAMS, C., "Ouidah's Door of Return: Diaspora Tours Are Still Tourism"; e para uma visão geral útil, consulte Araujo, A. L. (2018), "Tourism and Heritage Sites of the Atlantic Slave Trade and Slavery."

${ }^{5} \mathrm{Um}$ ensaio da história do tráfico de escravos francês e do aparato colonial ligando a Senegâmbia à Louisiana, o Caribe e o Brasil, e uma cobertura dos mais conhecidos monumentos da escravidão da África Ocidental e suas controvérsias, estão além da intenção deste artigo, mas o/a leitor interessado pode começar com esta amostra: ARAUJO, A. L. (2010). Public memory of slavery: Victims and perpetrators in the South Atlantic; THIAW, Ibrahima (2011), "Slaves without Shackles: An Archaeology of Everyday Life on Gorée Island, Senegal"; NORTHRUP, D. (2003), "New Evidence of the French Slave-Trade in the Bight of Benin"; MILLER, C. L., (2007), The French Atlantic Triangle; e FLEMING, C. M. (2017), Resurrecting slavery: Racial legacies and white supremacy in France. Sobre Gorée, arte-historicamente, veja HINCHMAN, M., (2015), Portrait of an Island: The Architecture and Material Culture of Gorée, Sénégal, 1758-1837. Digno de nota, o município de Goree em julho de 2020 mudou o nome de "Praça da Europa" para "Praça da Liberdade e Dignidade Humana", após os protestos liderados pelo Movimento Vidas Negras Importam (Black Lives Matter) na sequência da morte de George Floyd.
} 
Ou são, como diz Ian Grandison, "um conjunto de relações espaciais"? ${ }^{6}$ As imagens acima, e as duas que se seguem, extraí de um conjunto de fotos, parte de uma extensa foto-ensaio. As fotos foram reunidas, ao longo de quatro anos, no que caracterizei como caminhadas epistêmicas. aprender caminhando, por territórios tão pequenos como um jardim de um quintal ou parque da cidade, e tão grandes quanto uma extensão total de mangue, um bairro ou uma fazenda enorme. Na maioria das vezes, são fotografias de espaços e lugares, não de pessoas, e isso é de propósito, uma prática amadora acidental, que agora se tornou uma preferência. É a minha maneira falível de tentar olhar de perto, mas não agressivamente, em toda África e a diáspora; para capturar exemplos de criadores de mundos, mas não inadvertidamente congelar seus espíritos; de me forçar a imaginar os sentimentos e as ações dos humanos, mesmo quando não há nenhum no enquadramento. Este, então, é um projeto contínuo de tirar fotos, ouvir, escrever e re-escrever, mas teve um início definido. No Brasil - em Salvador! Deixe-me refazer alguns dos meus passos, não em ordem linear ou cronológica, mas sim como os fluxos, para frente e para trás, de pessoas, práticas e ideias diaspóricas ...

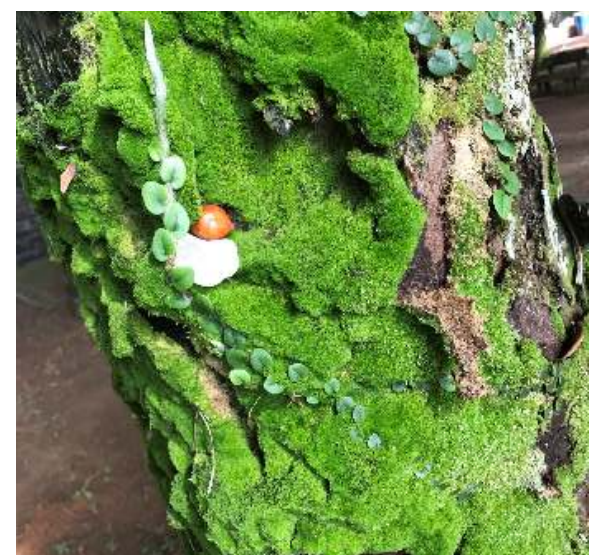

Dendê e os Ancestrais, Jaguaripes, Salvador da Bahia, Brasil, 2018.

\footnotetext{
${ }^{6}$ Grandison, K.I., (1999), "Negotiated Space: The Black College Campus as a Cultural Record of Postbellum America." Grandison está se referindo à paisagem, não à paisagem marinha, mas mesclo sua visão aqui com a de numerosos pensadores quilombolas (quilombolas) que argumentam persuasivamente que os cursos de água, a terra, a cultura e as relações sociais constituem uma unidade inseparável de prática, luta e análise: o território. Veja Augusto, G. (2016), "Transnacionalismo Negro: A Encruzilhada de Amefrican@s."
} 
Em Angola, Brasil, Benin e Nigéria, os caroços de dendê são importantes como fonte de um óleo vegetal rico em Vitaminas A e $\mathrm{K}$, usados numa variedade de pratos e medicamentos naturais. Quando morei em Angola durante a guerra, galinha com mwamba de dendem (frango ao molho de óleo de palma) sempre foi uma das minhas refeições favoritas, aos sábados. No Brasil, mais recentemente, fiquei muito feliz em encontrar o dendê (grafia brasileira) como ingrediente essencial em tantos pratos clássicos baianos. Mas o que realmente coloca o caroço de palma vermelho e seu óleo também no reino dos Ancestrais - como a cor branca - é seu uso em muitos aspectos do culto nas religiões afro-brasileiras e noutros lugares nas Américas. Em uma caminhada num bosque de dendenzeiros em Jaguaripes, Bahia, encontrei tanto o caroço caído quanto a pedra branca translúcida, nesta foto, lado a lado no chão, sob uma das palmeiras - então coloquei os dois entre as epífitas naquela mesma árvore.

E no passado? Pois bem, na região do Recôncavo, durante séculos repleta de plantações de cana-de-açúcar, os escravizados também mantinham roças (campos de provisões) onde cultivavam mandioca para si, e para uma cidade faminta. Lá eles também plantaram árvores de dendê - o suficiente para criar o que foi descrito como uma "paisagem de dendê" distinta, que se estende ao sul de Jaguaripes até Ilhéus, ao longo da costa baiana (OFFEN, 2018, p. 515). Numa paisagem tão vermelha e preta, eu imagino, o sagrado e o mundano devem ter se misturado, e uma árvore plantada por uma cativeira pode ter desempenhado muitos papéis.

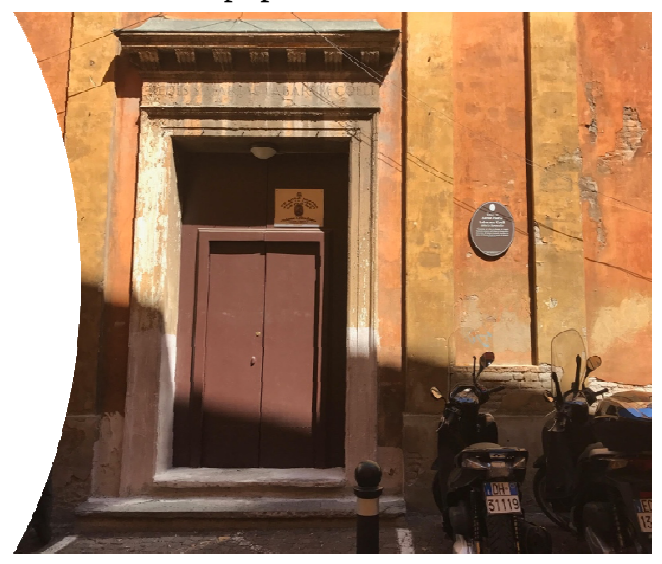

Adoração dupla. Igreja copta de Eritréia Kidane Mhret (na igreja de Santa Maria Labarum Coeli), Bolonha, Itália, Julho de 2017. 
Em frente ao pequeno apartamento onde ficamos alguns dias no centro de Bolonha, passei muitas vezes, em caminhadas de manhã cedo, por esta velha igreja, com seu exterior polido em terracota lindamente sombreado. Motocicletas elegantes sempre estavam estacionadas na frente, embora eu nunca tenha visto nenhum dos motoristas entrar ou sair. A minúscula placa oval do lado de fora da igreja proclamava sua história de séculos, mas a placa impressa, muito mais recente, logo acima da porta dobrava seu significado como espaço. Os eritreus constituem o segundo maior grupo de refugiados na Europa, e constituem uma proporção significativa dos que ainda vêm para a Europa. Um novo espaço político aquoso emergiu, o Mediterrâneo Negro, logo além das costas deste continente assolado por inseguranças e infortúnios neo-liberais para todos, exceto suas elites e os partidos de extrema direita. ${ }^{7} \mathrm{Na}$ Itália e em outras partes da Europa, há apreensão com o envelhecimento da população, mas também medo dos imigrantes da Europa Oriental, Oriente Médio e, principalmente, da África. $\mathrm{Ou}$, dito de outra forma, não estamos falando aqui da infame Passagem do Meio Atlântico, mas de uma kalunga, "um cemitério onde corpos pretos e pardos transitam por uma passagem hostil e mortal” (GUESMI, 2018, p. 1). Este emaranhado de eritreus e italianos não é realmente novo, alguns observadores apontam, apesar da negação, por alguns europeus, de qualquer conexão entre colonialismo, escravidão e os trágicos eventos de hoje, em suas costas. Como nota o crítico de arte Ismail Einashe numa resenha da recente exposição itinerante "Ressignificação": ${ }^{8}$

De 1880 a 1940, a Itália foi a potência colonial na Somália, Líbia e Eritreia e, em 1936, a Etiópia foi ocupada pelo estado fascista de Benito

\footnotetext{
7 O termo foi cunhado pela primeira vez pela acadêmica italiana Alessandra Di Maio, da Universidade de Palermo, que postulou em uma conferência da NYU [Universidade de Novo lorque] sobre a Black Italy [Italia Negra], que "enfoca a proximidade que existe, e sempre existiu, entre a Itália e a África, separadas [ ...] Mas também unidas pelo Mediterrâneo [...] e documentadas em lendas, mitos, histórias, até mesmo nas tradições culinárias, nas artes visuais e na religião. "Veja RAEYMAEKERS, T., "The Racial Geography of the Black Mediterranean, "2017.

8 Em EINASHE, I., "Reading the 'Black Mediterranean' through Europe's Migrant Crisis, " Frieze. Com curadoria do dramaturgo nigeriano e professor da Universidade de Novo lorque, Escola Tisch, Awam Amkpa, a exposição exibida na Zisa Zona Arti Contemporanee de Palermo representou corpos africanos na arte europeia, desde a época clássica greco-romana até o século 21, por meio de obras de vários artistas.
} 
Mussolini. Mas, na Itália, a cobertura da mídia sobre a migração tende a repetir que "essas pessoas" vieram do nada e sem motivo aparente. Mussolini chamou a cidade de Asmara, na Eritreia, "La Piccola Roma" - a pequena Roma da África, e encorajou os italianos a se estabelecerem lá, já que a cidade foi deliberadamente dividida em zonas raciais separadas.

Alternando cultos católicos e coptas na (ainda?) "Cidade Vermelha" de Bolonha, no epicentro desse novo / velho espaço, a igreja parcialmente sombreada parecia marcar um cosmos compartilhado de religiões nãoantagônicas, e pacificamente oscilantes. Mas também parecia sinalizar uma geografia urbana fugitiva, uma diáspora buscando construir, em seu deslocamento, uma nova pertença negra como os afro-italianos, em desafio à crescente violência anti-negra. ${ }^{9}$ Quem sabe quais serão seus contornos e fronteiras nos próximos anos?

\section{Coda}

Dispersos com respeito a tempo e lugar, os exemplos visualizados no meu foto-ensaio manifestam e compartilham certos atributos complexos da práxis contemporânea e do estudo de geografias, paisagens e arquitetura negras. Eu gostaria de propor que as paisagens, geografias e arquiteturas que estou estudando, demonstrem caracteristicas próprias - uma coda: móveis, gráficos, combinação do material e do imaterial, combinação "artearquitetura" e desafiadoras, ou sejam contrárias às expectativas ou imposições. Quero afirmar que esta coda pode oferecer premissas geradoras para uma reflexão crítica adicional, na intersecção de raça, espaço e cultura, justapondo, em vez de comparar, diferentes espaços em África e na Diáspora. Se assim for, esta reflexão continuará e se expandirá em caminhadas epistêmicas solitárias e coletivas, abrindo novos pontos de interrogação.

\section{Referências}

Adams, Chanelle. Ouidah's Door of Return: Diaspora Tours Are Still Tourism, The Funambulist, no. 17, May-June, p. 4-7, 2018.

${ }_{9}$ Para uma perspectiva renovadora de uma geógrafa feminista negra, consulte HAWTHORNE, C., "In Search of Black Italia: Notes on race, belonging, and activism in the black Mediterranean." 
Araujo, Ana Lucia. Public memory of slavery: Victims and perpetrators in the South Atlantic, Amherst, N.Y: Cambria Press, 2010.

Araujo, Ana Lucia. Tourism and Heritage Sites of the Atlantic Slave Trade and Slavery, em Dean, D. M. (Ed.), A companion to public history, Hoboken, NJ: Wiley-Blackwell, p. 277-288, 2018.

Augusto, Geri. Transnacionalismo Negro: A Encruzilhada de Amefrican@s. Revista Da Faeeba-Educac ão E Contemporaneidade, April 27, v. 25, no. 45, p. 25-38, 2016.

Bledsoe, Adam, Wright, Willie, \& Eaves, Latoya (January 01, 2020). Black Geographies, em Encyclopedia of Human Geography; p. 347-350, Elsevier Ltd.

Einashe, Ismail, (2018), Reading the 'Black Mediterranean' through Europe's Migrant Crisis, Frieze, 29 October. Disponível em: https://frieze.com/article/reading-black-mediterranean-through-europesmigrant-crisis, acesso 26/07/2019.

Fleming, Crystal. Resurrecting slavery: Racial legacies and white supremacy in France, Philadelphia: Temple University Press, 2017.

Grandison, Kenrick Ian. Negotiated Space: The Black College Campus as a Cultural Record of Postbellum America, American Quarterly, vol. 51, no. 3, September, p. 529-579, 1999.

Guesmi, Haythem. The Black Mediterranean and the limits of liberal solidarity, Africa Is a Country, August 30, 2018. Disponível em: https://africasacountry.com/2018/08/the-black-mediterranean-and-thelimits-of-liberal-solidarity, acesso on 26/07/19.

Hawthorne, Camilla. In Search of Black Italia: Notes on race, belonging, and activism in the black Mediterranean, Transition, January 01, issue 123, p. 152174, 2017.

Hinchman, Mark. Portrait of an Island: The Architecture and Material Culture of Gorée, Sénégal, 1758-1837, Lincoln: University of Nebraska Press, 2015.

McKittrick, Kathrine \& Woods, Clyde. Black geographies and the politics of place. Cambridge, Mass: South End Press, 2007.

Miller, Christopher. The French Atlantic Triangle: Literature and Culture of the Slave Trade, Durham, NC: Duke University Press, 2008.

Northrup, David. New Evidence of the French Slave-Trade in the Bight of Benin, Slavery \& Abolition, December 01, v. 24, no. 3, p. 61-81, 2003. 
Offen, Karl. Environment, Space, and Place: Cultural Geographies of Colonial Afro-Latin America. In Fuente, Alejandro, and Andrews, George R.. Afro-Latin American Studies: An Introduction. New York: Cambridge University Press, p. 486-534, 2018.

Raeymaekers, Timothy. "The Racial Geography of the Black Mediterranean," The Dreaming Machine, November 30, 2017. Disponível em: http://www. thedreamingmachine.com/the-racial-geography-of-the-blackmediterranean-timothy-raeymaekers , acesso 26/07/2019.

Revista da ABPN, (Abr 2020), EDIÇÃO ESPECIAL-Caderno Temático “Geografias Negras" v. 12.

Thiaw, Ibrahima. "Slaves without Shackles: An Archaeology of Everyday Life on Gorée Island, Senegal," in Lane, Paul and MacDonald, Kevin (Eds.), Slavery in Africa: Archaeology and memory, Oxford: Published for the British Academy by Oxford University Press, p. 147-165, 2011.

Recebido em 20 de novembro de 2020

Aceito em 15 de dezembro de 2020 